\title{
Optical and Near-Infrared Imaging of Young Binary Star Environments
}

\author{
François Ménard \\ Canada-France-Hawaii Telescope Corporation, PO Box 1597, Kamuela, \\ HI 96742, USA
}

Karl Stapelfeldt

MS 183-900, Jet Propulsion Laboratory, California Institute of Technology, Pasadena CA 91109, USA

\begin{abstract}
We review recent imaging results on the circumstellar matter of young binaries obtained using the Hubble Space Telescope (HST) and by PUEO, a ground-based adaptive optics (AO) system operating at CFHT. In the area of circumbinary disks, new results for GG Tau help form a more complete picture of this prototype system. However, HST images of the UY Aur ring indicate a more complex system than first thought. A new example of a circumstellar disk in a multiple star system has been found in HV Tau, joining HK Tau in this category. Examples of envelopes, outflow cavities, and jets are shown for several young binaries. About half of the nearby young binaries in our survey possess some kind of local nebulosity at optical or near-IR wavelengths.
\end{abstract}

\section{Introduction}

High dynamic range diffraction limited images are now (almost) routinely available in the optical and near-infrared, thanks to the Hubble Space Telescope and to ground-based adaptive optics systems. These technological advances provide the necessary resolution to observe features on 0.11 (10-15 AU) scales in nearby star forming clouds. This resolution is well matched to the size scales of accretion disks, typically a few hundred AU, and for studies of young binaries themselves whose separation distribution peaks near $30 \mathrm{AU}$. Using this capability, important progress is taking place in the study of young binary star environments.

At optical and near-infrared wavelengths HST and AO detect scattered light from circumstellar/circumbinary dust. The dust opacity is high at these wavelengths, if the dust particles have properties similar to interstellar grains. This means that tenuous circumstellar structures are detectable: a very small amount of dust is enough to produce a detectable reflection nebula. The large dust opacity also means however that accretion disks should be very optically thick, possibly impeding our capacity to probe their internal structure in detail. Our experience shows that an important complication arises from the proximity of the bright core of the PSF, the unresolved image of the bright central star. Reaching a large enough contrast (i.e., dynamic range) to distinguish the faint and extended nebulae from the PSF often proves very challenging. 
Our observing programs are mostly based on large HST snapshot imaging campaigns. The targets were selected to maximize the probability of disk detection. Criteria like resolved molecular gas disks, millimeter continuum emission, optical nebulosity, large polarisation, strong $\mathrm{H} \alpha$ emission, and location in nearby clouds were used. More than 100 young stars have been imaged so far with WFPC2 on HST. A large number of these were also observed with AO in the near-infrared.

In the following sections we will present our HST and AO results for some of the 40 binaries that we observed. Two cases of circumbinary disks will be presented, two cases of circumstellar disks in binary systems, and finally examples of circumbinary envelopes and mass outflows are shown from our survey.

\section{Circumbinary Disks: GG Tau and UY Aur}

On average, young binaries with separations in the range 1-100 AU have lower dust millimeter continuum emission (Jensen et al. 1996; Osterloh \& Beckwith 1995; Dutrey et al. 1996). The number of objects with resolved emission, either the dust continuum or the moleculer lines, remains deceivingly low. In the case of UZ Tau W, the continuum appears slightly extended with respect to the beam, and the velocity gradient along the major axis suggests rotation of a disk (Jensen, Koerner \& Mathieu 1996). But to our knowledge, only in two cases have circumbinary disks been resolved with more than 2 or 3 resolution elements by radio interferometers. These disks have also been resolved in scattered light at shorter wavelengths. They are found around GG Tau and UY Aur.

\subsection{UY Aur}

UY Aur is a binary made of two Classical T Tauri stars of spectral types K7 and M2 (Duchêne et al. 1999) separated by $0^{\prime \prime} .9$ (125 AU). Both stars have a nearinfrared excess, suggesting that they each possess circumstellar disks. Interesting to us, circumbinary molecular CO gas was discovered by Duvert et al. (1998). Its kinematics is compatible with Keplerian rotation. The millimeter continuum emission from the dust in the circumbinary disk is not detected however, but Close et al. (1998) secured the first near-infrared images of the scattered light (by dust) in the same circumbinary ring using adaptive optics at the CanadaFrance-Hawaii Telescope. Their image is presented in Figure 1b). Figure 1a) presents the optical counterpart from HST/WFPC2.

On the HST image (Fig. 1a), two components are identified in the circumstellar environment: a bright and narrow extended filament, and a more diffuse, broad patch of nebulosity located at the South-East end of the filament. The filament appears to overlap the more diffuse patch to the SE. Artifacts are present and are discussed in the caption, but are located well inside the circumbinary ring and thus have little effect on our ability to study it.

The near-infrared AO image (Fig. 1b) shows the same basic structures as seen in the HST image. Noticeably however, the nebulosity appears more clumpy. This is partly because of artifacts (diffraction spikes) but also because the SE broad patch seen with HST is not resolved as sharply in the AO image.

The filament seen offset SW of the binary in Fig. 1 extends along a position angle quite close to that of the rotating gas disk detected in ${ }^{13} \mathrm{CO}(2 \rightarrow 1)$ with the IRAM interferometer, namely $135^{\circ}$ (Duvert et al. 1998). It also extends over 

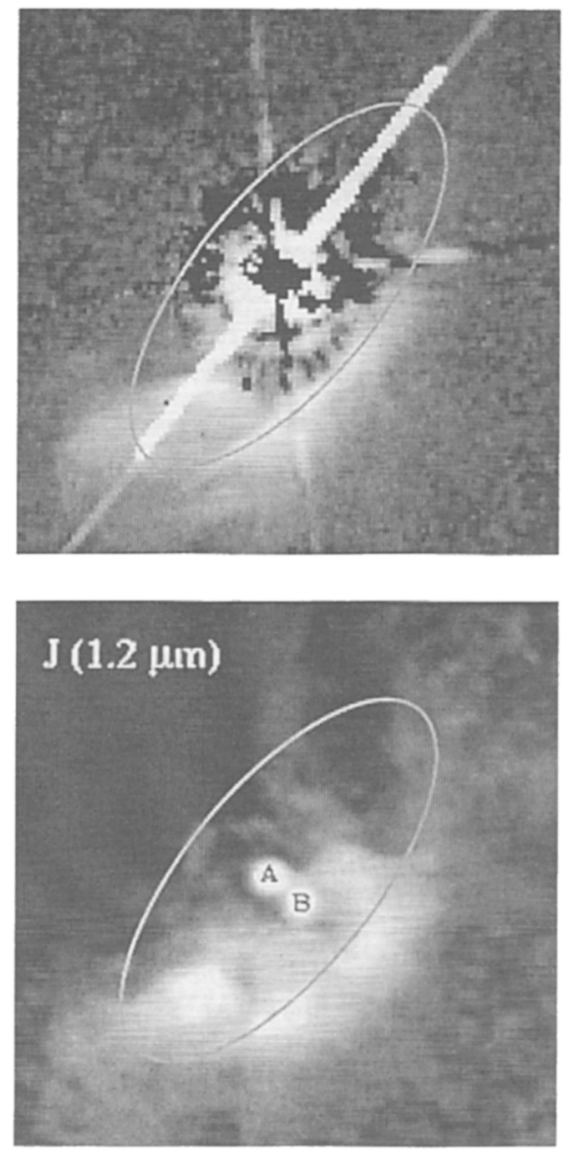

Figure 1. A) (top panel): HST image of the binary UY Aur obtained with WFPC2 (F606W filter). Two PSFs have been subtracted from the image. Imperfections in the PSF subtraction can be seen as negative (black) regions close to the central stars. The primary is saturated and the CCD "bleeding" trail is seen in diagonal, as the image was rotated.

B) (bottom panel): Nearinfrared J-band adaptive optics image of UY Aur. This is the first image of the circumbinary ring in scattered light. The image is deconvolved. Artifacts are also visible close to the center, due mostly to the diffraction spikes. North is up and East to the left on both images. An ellipse aligned with the NW trending filament, suggesting the geometry of the ring, is overplotted on each image.

roughly the same distance across the central objects, $r \sim 800 \mathrm{AU}$, indicating that the scattered light structure most likely to be associated with the gas disk. This indicates that the ring contains a dust component despite its non-detection in the millimeter continuum. The nature of the SE patch is much less clear; it seems discontinuous from the SW filament, and thus it is not obvious whether it is also associated with the disk. Close et al. (1998) assumed that both structures traced the inner edge of the circumbinary ring and estimated its inclination to $i=42^{\circ}$ by ellipse fitting.

A new fit to the disk inclination using the filament alone gives $i=64^{\circ}$. We estimate the range of acceptable values to $59^{\circ}<i<65^{\circ}$. This implies a significantly more edge-on disk than suggested by Close et al. (1998). The result of our fit, an ellipse, is superimposed on Figs. 1a) \& b). We believe this result is closer to reality and we present arguments below.

Close et al. (1998) measured the contrast between the nebulosity on the NE and SW sides of UY Aur, respectively the back- and front-side of the circumbinary disk. The contrast increases up to a value of $\sim 8$ at shorter NIR wavelengths, at $1.2 \mu \mathrm{m}$. The HST images of Fig.1a shows the contrast to be at least 15 (or $3^{\text {mag }}$ ) at $0.6 \mu \mathrm{m}$. Ménard et al. $(1999,2000$ in prep.) argue that 
the larger value of inclination explains naturally this large contrast. It is a direct consequence of the inefficiency of ISM-sized spherical grains to backscatter photons at large angles. Small grains are naturally forward-throwing, and large disk inclinations naturally lead to large contrasts when illuminated by a central source. The inclination of $\sim 45^{\circ}$ estimated by Close et al. (1998) cannot explain the large contrast observed in the optical except with very exotic grain mixtures.

The inclination of the circumbinary ring has a significant effect on the interpretation of the CO kinematics. Using the $42^{\circ}$ inclination derived from the AO images, Duvert et al. (1998) concluded that the highest radial velocity gas had to be located interior to the circumbinary ring. This result was somewhat surprising, since the central hole should be dynamically cleared by gravitational perturbations from the binary. With the more edge-on ring inclination implied by the HST image, the CO kinematics can be reinterpreted and the presence of gas interior to the ring is no longer required. However, the presence of material within the "gap" is not ruled out by our observations.

The nature of the broad SE patch is unclear at present but it can be shown that it is coincident with a peak of $\mathrm{CO}$ emission that is independent kinematically from the Keplerian disk. This new result supports our suggestion that there are two independent components in the circumbinary environment of UY Aur.

\subsection{GG Tau}

This fascinating object has been the center of much attention since the discovery of its massive circumbinary ring of dust and gas. See also the contributions by McCabe (2001) and Guilloteau (2001) in this book for example.
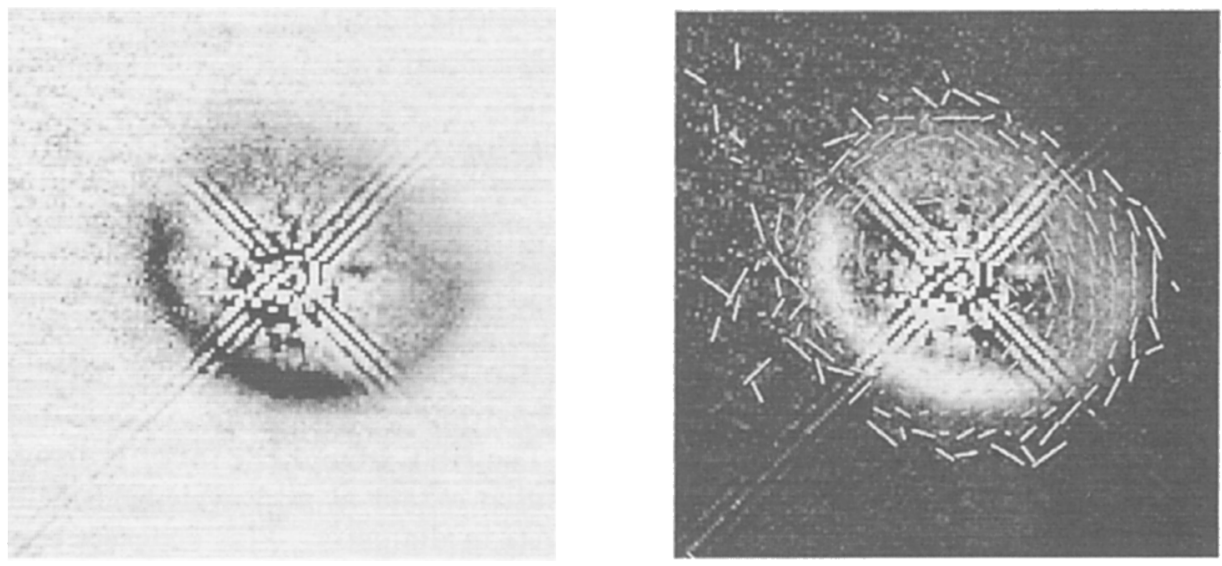

Figure 2. HST/NICMOS $1 \mu \mathrm{m}$ images of the GG Tau circumbinary ring. A) (left panel): Intensity image. B) (right panel): Same image as A) with linear polarisation superposed. Images are from Silber et al. (2000). The diffraction spikes are oriented along the cardinal directions. North is toward the bottom left corner, East toward bottom right corner. The gap in the disk ("dark sector") is located at PA $\sim 275^{\circ}$ in the equatorial system. It is located just below the top-left diffraction spike. 
Our images complete the existing data sets and allow to settle a few issues. Optical images, obtained with HST/WFPC2 by Krist et al. (2000), and nearinfrared $1 \mu \mathrm{m}$ image and linear polarisation map, obtained with HST/NICMOS by Silber et al. (2000), provide an accurate view of the scattered light by the disk and hence of the dust distribution and properties.

From our images, see Fig. 2, the brightness distribution in the circumbinary ring appears smooth, and there is no evidence for the radial "spokes" suggested by Roddier et al. (1996). Their images had lower signal-to-noise ratio. Our deeper images, obtained with two different instruments and two different roll angles of the spacecraft (i.e., the diffraction spikes are therefore not at the same place on the two sets of images) allow us to make that claim. Our images further reveals a "dark sector" located just below the top-left diffraction spike on Fig.2 and covcring $\sim 15^{\circ}$ in azimuth. The origin of this apparent gap in the brightness distribution is unclear at the moment.

The ring appears red with respect to the central stars, and the front/back intensity ratio is about 3.3 at $1 \mu \mathrm{m}$, similar to the value found by Roddier et al. (1996). Furthermore, the linear polarisation measured in that band is large on the back-side ( $\mathrm{P} \sim 50 \%)$ and lower on the front side ( $\mathrm{P} \sim 20 \%)$. Assuming an inclination of $37^{\circ}$, these numbers firmly imply that the dust grains are small, well into the Rayleigh scattering regime at $1 \mu \mathrm{m}$. The red color of the disk is puzzling however and calls for severe extinction of the photospheric light by inner circumstellar disks. Even more intriguing is the lack of symmetry of the brightness distribution about the projected minor axis. This is potentially an effect of illumination by a binary instead of a single star. Krist et al. (2000, in prep.) suggest that this effect may also be explained if the ring has some intrinsic eccentricity instead of being perfectly circular (as has been generally assumed). Further models are needed to verify this hypothesis.

\section{Circumstellar Disks in Binary Systems}

The classical T Tauri binary HK Tau was imaged nearly simultaneously with HST/WFPC2 and with AO at the Canada-France-Hawaii Telescope (Stapelfeldt et al. 1998a), and with Keck speckle imaging (Koresko 1998). The three techniques produce complementary results and provide extended wavelength coverage necessary for understanding the circumstellar material distribution. These results are interesting as the first well-resolved images of a circumstellar disk around one component of a binary system. The HST I-band (F814W) is shown in Fig.3a, left panel. The disk has a very similar extent at all wavelengths from $600 \mathrm{~nm}$ to $2200 \mathrm{~nm}$, namely $\sim 1$ 1.5 or 210 AU in diameter. This suggests that the disk's edges are likely to be fairly sharp. A second example of resolved circumstellar disk in a young multiple star system has now been discovered: HV Tau C (Fig. 3b; Monin \& Bouvier 2000; Stapelfeldt et al. 2000, in prep.). Here the disk diameter is even smaller, only 170 AU. The sizes of these two young disks are among the smallest yet measured in scattered light, with radii $1 / 7$ $1 / 3$ that of the projected separation of the components. This suggests that there may be a link between the small disk sizes and their presence in a multiple star environment; the disk outer radii may be truncated by tidal interactions with the primary star. It is too early to make firm conclusions (the binary orbital 


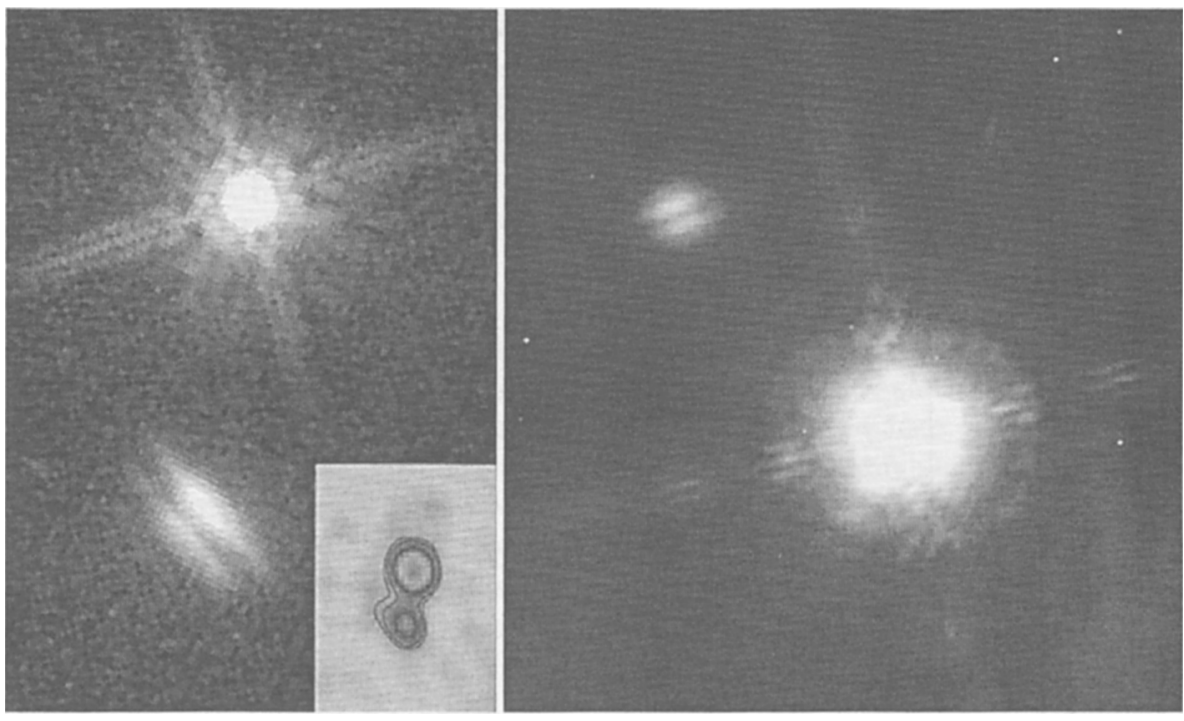

Figure 3. Images of two circumstellar disks in binary stars. On both panels North is up and East to the left. A) (left panel): HST/WFPC2 image of the HK Tau system. The separation is 2.4". HK Tau B, to the south, is nebulous and extended. The insert is a map obtained with the IRAM Plateau de Bure Interferometer of the dust thermal emission at $1.4 \mathrm{~mm}$. B) (right panel): CFHT/PUEO Adaptive Optics image of HV Tau. The companion, 4" to the NorthEast, is also nebulous and extended. In both cases no central star is detected and the dark lane crossing the companions is interpreted as a fine example of a disk seen edge-on. The image is from Stapelfeldt et al. (2000, in prep.).

elements are not yet known for either system), but HK Tau and HV Tau are strong candidates for this long-known theoretical possibility.

A dark lane is clearly seen at the secondary in both systems shown in Fig. 3, and one can safely infer that each is a nearly edge-on disk. Numerical simulations of the scattered light from the disk give $i=5^{\circ} \pm 1^{\circ}$ for HK Tau B (Stapelfeldt et al. 1998) and $i<6^{\circ} \pm 1^{\circ}$ for HV Tau C (Monin \& Bouvier 2000). The central star is not seen in either case, even at $2.2 \mu \mathrm{m}$ where the extinction is 10 times smaller than at $550 \mathrm{~nm}$. Given our detection limit for a point source toward HK Tau $B$, we estimate that the extinction is $A_{v}>50$ magnitudes in the plane of this disk, in line with the idea they are optically thick.

The nearly edge-on orientation is very favorable to study the scale height of the dust distribution in the disk plane. That possibility was discussed and tested extensively by Burrows et al. (1996) in the case of HH 30. Models for HK Tau B are presented in Stapelfeldt et al. (1998). They were obtained for single scattering by dust grains uniformly mixed within a gaseous disk whose parametrized surface density $\Sigma(r)=\Sigma_{0}\left(r / r_{0}\right)^{p}$ and with a Gaussian vertical density profile $\rho(z)=\rho_{0} \exp \left[-z^{2} / H(r)^{2}\right]$. This vertical profile is valid for a vertically isothermal, hydrostatic, non-self-gravitating disk. A parametrized scale 
height $H(r)=H_{0}\left(r / r_{0}\right)^{\beta}$ was further assumed. We adopted an outer radius of 105 AU, consistent with Fig.3. A $\chi^{2}$ minimization procedure was used to solve for the disk parameters. The normalization $H_{0}$ of the scale height is $3.8 \mathrm{AU}$ at $r=50 \mathrm{AU}$. This number is well constrained. Hence, the disk of HK Tau B is significantly flatter than HH 30's where $H_{0}=15.5 \mathrm{AU}$ at $r=100 \mathrm{AU}$. Presented differently, at $50 \mathrm{AU}$ the ratio $H / r=0.08$ for HK Tau B but 0.12 for HH 30 .

The mass of an edge-on disk is directly related to the "thickness" of the dark lane. For HK Tau B, we estimate a total disk mass of $\sim 10^{-4} \mathrm{M}_{\odot}$ in dust and gas, if the grains have interstellar properties. This is small with respect to the minimum mass solar nebula and small with respect to the mass implied by the $1.3 \mathrm{~mm}$ continuum flux density $(40 \mathrm{mJy}$, Osterloh \& Beckwith 1995). The expected disk mass from this mm-flux is roughly 40 times larger. Ménard et al. (2000b, in prep.) report that $2 / 3$ of the continuum flux from the system comes from the primary, partially solving the mismatch in masses and clearly indicating that both stars in the system have circumstellar disks. For HV Tau C, preliminary estimates suggest a total disk mass of $\sim 4 \times 10^{-4} \mathrm{M}_{\odot}$, with the same assumptions regarding the dust and gas. The model matches the dust lane thickness over the range $0.6<\lambda<2.2 \mu \mathrm{m}$ (Stapelfeldt et al. 2000, in prep.).

The results presented above are not exhaustive. They were used mainly to demonstrate the power of high-angular resolution imaging and the complementarity between ground-based and spaced-born multiwavelength approaches to study the structure of accretion disks. Admittedly, scattered light images of disk seen nearly edge-on are very sensitive tools to examine the circumstellar environment of pre-main sequence objects. But such low-mass disks become more difficult to detect if seen more pole-on. A pole-on disk was imaged around the single T Tauri star TW Hya (Krist et al. 2000). However, this object lies at only half the distance of most of the T Tauri stars we have studied, and this difference may have been crucial for the detectability of its disk. More research, especially with larger millimeter interferometers will help further our knowledge of the disks around young stars.

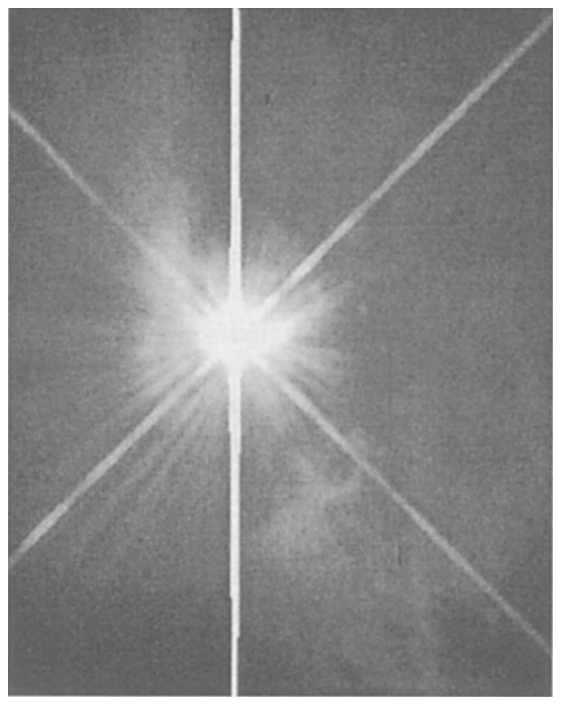

Figure 4. A deep HST Planetary Camera image of $\mathrm{T}$ Tauri at a wavelength of 0.6 micron, shown in $\log$ stretch. The field of view is $15 \times 18 \operatorname{arcsec} ; \mathrm{N}$ is toward the upper left and $\mathrm{E}$ to the lower left. Only the primary star is directly seen, superimposed on an asymmetric nebulous arc passing from its upper left and across to the right. Irregular wisps of reflection nebulosity appear below and to the right of the primary. The vertical spike is a saturation artifact. 


\section{Envelopes, Outflows and Jets}

The number of envelopes we detected in our surveys is much larger than the number of disks for example, by 5 to 1 roughly. In this section we will therefore only show a few key examples and we will summarize our results at the end.

\subsection{T Tauri, the Prototype for Young Stellar Object Complexity}

Brightest member of the group of eleven that initially defined $\mathrm{T}$ Tauri stars as a class, T Tauri has become a prototype for complexity in young stellar object environments. It harbours all the signs of youth: Lithium in absorption, strong IRAS and millimeter emission, and a powerful outflow. It also has a luminous infrared companion ( $\mathrm{T}$ Tau South) which may itself be a binary (Koresko 2000). For a long time the reflection nebulosity associated to $\mathrm{T}$ Tau has been known for its variability. Initial HST images reported by Stapelfeldt et al. (1998b) suggest an asymmetric outflow cavity centered on the primary. A deeper HST image (see Fig. 4) shows still more complexity in the system, with "bubbles" apparently being ejected, wisps tracing the edges of cavities, and so on.

\subsection{Large Envelopes: The Case of CoKu Tau/1}
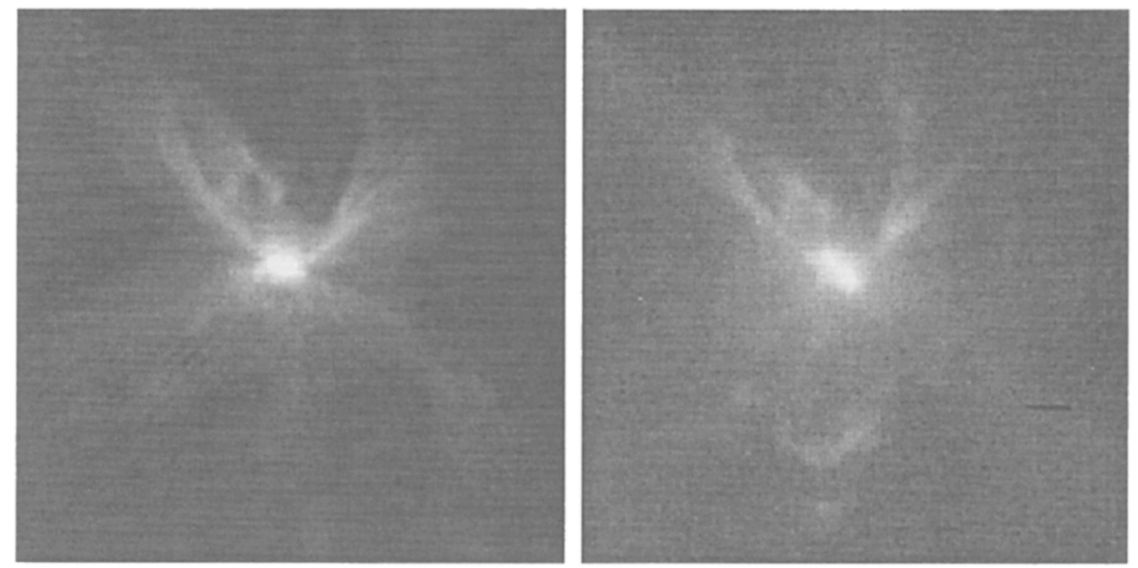

Figure 5. HST/NICMOS (left panel) and WFPC2 (right panel) images of CoKu Tauri/1. Images are from Padgett et al. (1999) and Stapelfeldt et al. (2001, in prep.) respectively. Four extended "wings" of reflection nebulosity trace the circumbinary outflow. cavity and can be seen in both images.

The case of CoKu Tauri/1 is particularly interesting in the scope of this contribution. The object itself is an 0. " 24 (34 AU) binary with the companion $\sim 1$ mag fainter than the primary. A large hourglass shaped reflection nebulosity traces a cavity evacuated by an outflow as suggested by Padgett et al. (1999) (Fig.5, left panel). It is the source of the small HH 156 bipolar jet, visible in Fig. 5 right panel. The kinematics of the jet suggests that the flow is nearly in the plane of the sky (Eislöffel \& Mundt 1998). In the HST/NICMOS image (Fig.5, left panel), the surface brightness goes through a minimum along a line 
that is perpendicular to the axis of the bipolar cavities. This can be interpreted as a "dark lane" caused by dust located in the equatorial plane, a circumbinary dust lane/disk? This picture is in agreement with the kinematics of the jets. The right panel shows the HST/WFPC2 image where the shock emission in the jet is visible. The outward proper motions are of order $180 \mathrm{~km} / \mathrm{s}$ (Eislöffel \& Mundt 1998), and the morphology of the jet knots suggest a wide opening angle for the outflow, of order $20^{\circ}$ The base of the outflow seems to indicate that the embedded companion is the probable source of the flow.

\subsection{Mass Loss: RW Aurigae \& XZ Tauri}

Other fine examples of ionised jets from young binaries are offered by RW Aur and XZ Tau. In the case of RW Aur, see Fig. 6, the image of this hierarchical triple unambiguously shows that the jet is driven by the primary. The secondary (itself a binary) is located 1."4 away ( 200 AU). This jet is in all aspects similar to other microjets driven by single T Tauri stars (Dougados et al. 2000). It has similar opening angle $\left(\sim 3^{\circ}\right)$, morphology, tangential and radial velocity. The jet width is resolved, at about $0 !^{\prime \prime} 2-0 !^{\prime \prime} 25$ (or 25-35 AU), as close as $56 \mathrm{AU}$ from the driving source. Density collimation must therefore occur on smaller spatial scales than this limit, in quantitative agreement with current MHD wind models (e.g., Cabrit, Ferreira \& Raga, 1999; Shang et al. 1998). This, and the previous results on CoKu Tauri/1, suggest that the jet driving mechanism is not disturbed significantly by the presence of a close (50-200 AU) companion.

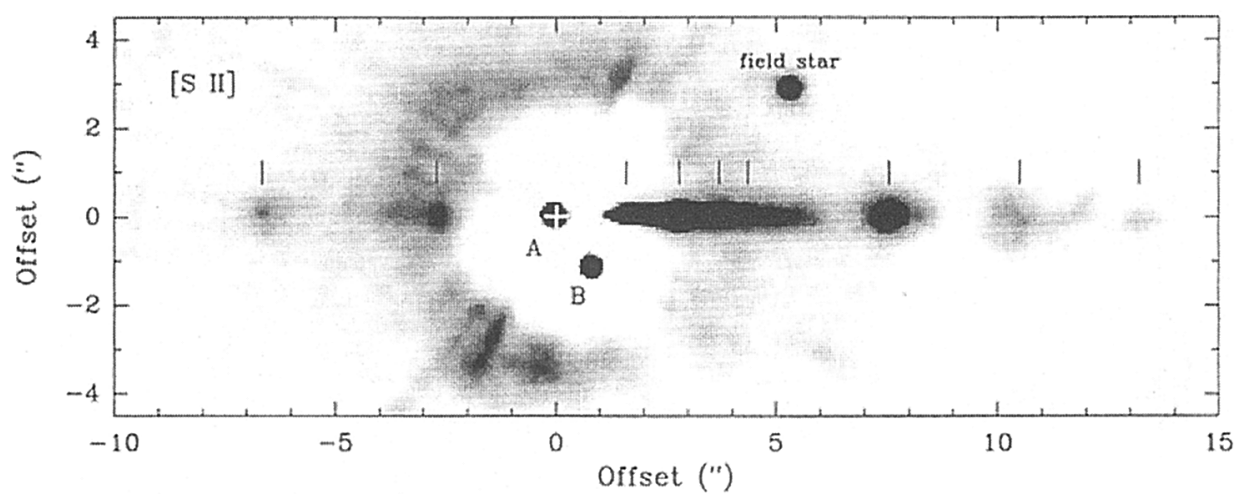

Figure 6. Optical image of the pre-main sequence binary RW Aur obtained at CFHT with PUEO. The micro-jet around RW Aur A is clearly visible in this [S II] $\lambda \lambda 6717 / 6731 \AA+$ continuum deconvolved map. The resolution (FWHM) is $0 ! 1$. This image is the combination of three coresaturated exposures (300 sec each) with two unsaturated images. The final dynamic range is $\sim 10^{6}(1 \sigma)$. The centroid position of RW Aur A is indicated by a cross.

The case of XZ Tau is more dramatic. This $0 .^{\prime \prime} 3$ (42 AU) binary (Haas et al. 1990) was literally caught in the act of blowing an outflow "bubble". The HST images of Krist et al. $(1997,1999)$ show a rapidly expanding "bubble" of shocked gas that is now 5" (700 AU) long. With an estimated proper motion of $\sim 145$ $\mathrm{km} / \mathrm{sec}$, they could trace back the ejection of the bubble to the year $\sim 1975$. The 
bubble has a complex internal structure. In that case we are possibly seeing the early stages in the evolution of an Herbig-Haro flow.

\section{Summary}

Many sources with interesting nebular environments have been identified and their structure studied with unprecedented resolution. Follow-up studies are under way. They will include optical and near-infrared complementary imaging, millimeter mapping, and variability studies.

In our HST snapshot surveys, nebulosity is detected in $50 \%$ of the young binaries, and in $35 \%$ of young single stars. At this point it is not clear how to account for the non-detections - there are many in systems with strong infrared and millimeter evidence for dust. Most likely, we are suffering from insufficient dynamic range in the data. The era of large telescopes either in space or corrected by high order $\mathrm{AO}$ promises to bring a clear view of many more young binary environments.

\section{References}

Burrows, C. J., Stapelfeldt, K. R., Watson, A. M., et al. 1996, ApJ, 473, 437

Cabrit, S., Ferreira, J., Raga, A. 1999, A\&A, 343, L61

Close, L. M., Dutrey, A., Roddier, F., Guilloteau, S., Roddier, C., Duvert, G., Northcott, M., Ménard, F., Graves, J. E., Potter, D. 1998, ApJ, 499, 883

Dougados, C., Cabrit, S., Lavalley, C., Ménard, F. 2000, A\&A, 357, L61

Duchêne, G., Monin, J.-L., Bouvier, J., Ménard, F. 1999, A\&A, 351, 954

Dutrey, A., Guilloteau, S., Duvert, G., Prato, L., Simon, M., Schuster , K., Ménard, F. 1996, A\&A, 309, 493

Duvert, G., Dutrey, A., Guilloteau, S., Ménard, F., Schuster, K., Prato, L., Simon, M. 1998, A\&A, 332, 867

Eislöffel, J., Mundt, R. 1998, AJ, 115, 1554

Guilloteau, S. 2001, this volume

Haas, M., Leinert, Ch., Zinnecker, H. 1990, A\&A, 230, L1

Jensen, E. L. N., Koerner, D. W., Mathieu, R. D. 1996, AJ, 111, 2431

Jensen, E. L. N., Mathieu, R. D., Fuller, G. A. 1996, ApJ, 458, 312

Koresko, C. D. 1998, ApJ, 507, L145

Koresko, C. D. 2000, ApJ, 531, L147

Krist, J. E., Burrows, C. J., Stapelfeldt, K. R., et al. 1997, ApJ, 481, 447

Krist, J. E., Stapelfeldt, K. R., Burrows, C. J., et al. 1999, ApJ, 515, L35

Krist, J. E., Stapelfeldt, K. R., Ménard, F., Padgett, D. L., Burrows, C. J. 2000, ApJ, in press

McCabe, C. E. 2001, this volume

Ménard, F., Stapelfeldt, K., Krist, J., et al. 1999, AAS, 194.6811

Monin, J.-L., Bouvier, J. 2000, A\&A, 356, L75

Osterloh, M., Beckwith, S. V. W. 1995, ApJ, 439, 288 
Padgett, D. L., Brandner, W., Stapelfeldt, K. R., Strom, S. E., Terebey, S., Koerner, D. 1999, AJ, 117, 1490

Roddier, C., Roddier, F., Northcott, M. J., Graves, J. E., Jim, K. 1996, ApJ, 463,326

Shang, H., Shu, F. H, Glassgold, A. E. 1998, ApJ, 493, L91

Silber, J., Gledhill, T., Duchêne, G., Ménard, F. 2000, ApJ, 536, L89

Stapelfeldt, K. R., Krist, J. E., Ménard, F., Bouvier, J., Padgett, D. L., Burrows, C. J 1998a, ApJ, 502, L65

Stapelfeldt, K. R. et al. 1998b, ApJ, 508, 736

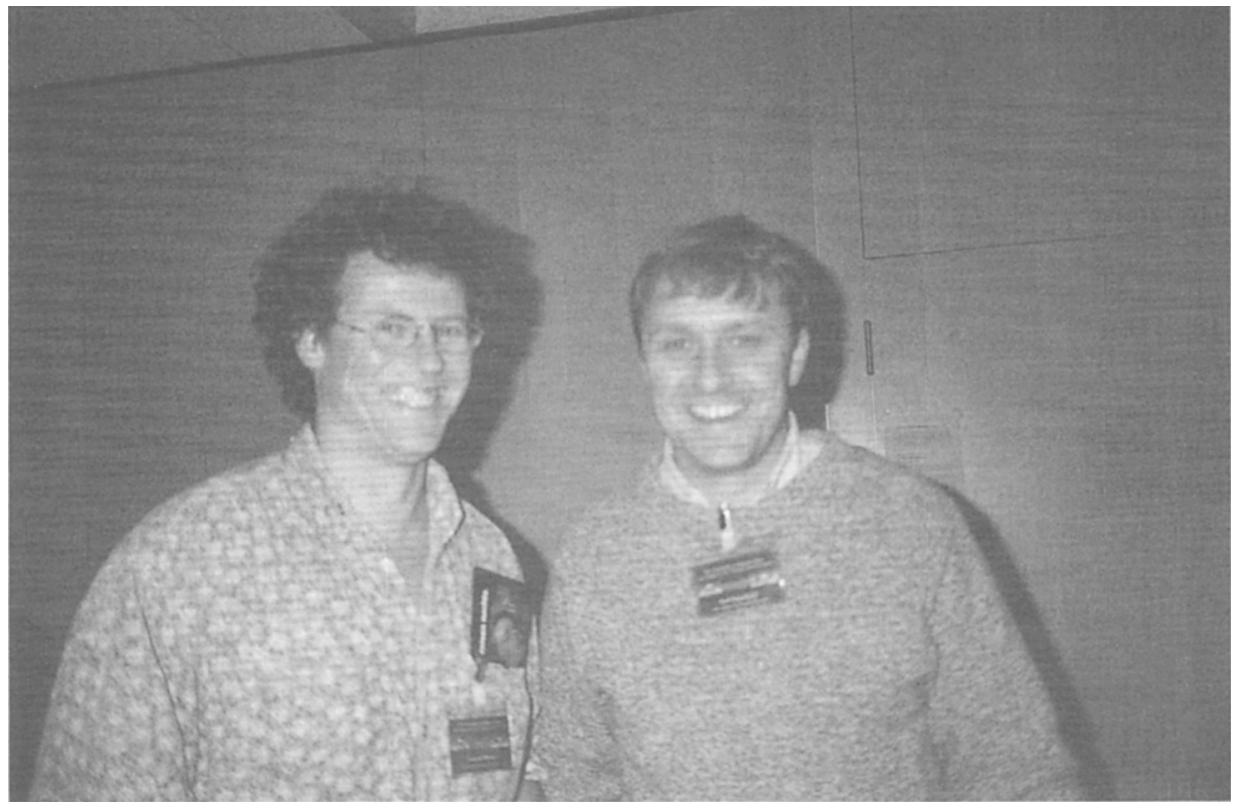

François Ménard, Karl Stapelfeldt 